\title{
Description of Multichannel Amplification in Semiconductor Optical Amplifier
}

\author{
Regular Paper
}

\author{
Sadiq Jaafar Kadhim¹, Ali Hadi Hassan² and Hassan Abid Yasser ${ }^{1, *}$ \\ 1 Iraq, Thi-Qar University, Science College, Physics Department \\ 2 Iraq, Al-Mustansyria University, Education College, Physics Department \\ *Corresponding author E-mail: hahmha@yahoo.com
}

Originally published in the International Journal of Radio Frequency Identification \& Wireless Sensor Networks, ISSN 1847-9812

(C) 2012 Kadhim et al.; licensee InTech. This is an open access article distributed under the terms of the Creative Commons Attribution License (http://creativecommons.org/licenses/by/3.0), which permits unrestricted use, distribution, and reproduction in any medium, provided the original work is properly cited.

\begin{abstract}
In this paper, a generalized equation of pulse propagation in semiconductor optical amplifier (SOA) under the effects of four wave mixing (FWM) has been derived. This equation is applicable for any wavelength division multiplexing system in presence of both interband and intraband effects. The equation is solved numerically regarding the primary conditions of the problem considering the amplitudes of the sideband pulses at the entrance of SOA are zero. The gain of low frequency pulses will be higher than the gain of the high frequency pulses, as well as the gain difference between different pulses will be reduced with the increasing of frequency detuning. On the other hand, the effects of saturation and the asymmetric gain will control the mixing efficiency of the sideband pulses generated on the left and right of active pulses depending on the power of the input pulses and the frequency detuning.
\end{abstract}

Keywords SOA, FWM, WDM system

\section{Introduction}

The use of semiconductor optical amplifiers (SOAs) as inline amplifiers has received renewed interest due to the penetration of optical networking into the metro market [1-3]. An SOA used as a receiver preamplifier is an elegant approach to optical pre-amplification since the SOA can be integrated with the photo detector. An SOA array can even be used as a compact channel equalizer where the wavelength channels can be equalized through gain adjustment on their individual amplifiers [4,5]. SOAs have already proven their worth in applications for high capacity transmission in metropolitan environments. Therefore, a compact SOA with the potential for mass production and the ability to be integrated into other passive distribution platforms is very attractive for the cost-sensitive metro market. In multichannel fiberoptic communication systems using wavelength division multiplexing (WDM) system [6-8], FWM gives rise to interference between channels, thus decreasing the channel quality. Impairments due to FWM play a role in pseudo-linear transmission systems $[9,10]$ as well as in parametric amplifiers. FWM products are of particular importance, as those products fall into the signal band. Each FWM product has different power and the total powers can be used to calculate the noise generated at each frequency [1113]. 
The determination of FWM products number is a classical problem, but the calculation of each power generated at a certain frequency is very important subject. WDM promises advantages for switching and routing as well as for transmission $[9,10]$. Optical crossconnects are currently being developed which can switch an entire wavelength from an input fiber to an output fiber. Particularly, simple and compact wavelength converters that help to avoid wavelength blocking. All-optical wavelength conversion has successfully been demonstrated with SOA devices exploiting the FWM [11].

Carrier density modulation and the associated nonlinear phenomenon of FWM affect the performance of amplifiers in two ways. First, the amplifier gain becomes different for different channels. As a result, the output channel powers become unequal even if all channels have the same power at the amplifier input. second some energy is transferred to new sidebands located on each side of the boundary channels as a result of FWM making the amplifier less efficient [12].

In this paper, we are presented a new analysis of FWM to describe the amplification of an arbitrary number of input fields in WDM system by including the combined effects of interband and intraband processes. This case is called as multichannel mixing. The FWM process affects the active channel gains as well as generates new sidebands beyond the boundary channels. We illustrate these effects by describing different systems. The analysis will be done numerically since the analytic one is impossible.

\section{Theoretical Treatment}

Consider a large number of modes with amplitudes $A_{-n}, \ldots . . A_{-1}, A_{o}, A_{1}, \ldots . A_{n}$ and angular frequency $w_{-n}, \ldots ., w_{-1}, w_{o}, w_{1}, \ldots . w_{n}$. The total electrical field be described as a set of discrete oscillating spectral modes

$$
\vec{E}(r, \tau)=\hat{e} U(x, y) \sum_{m=-n}^{n} A_{m}(z) e^{-i w_{m} \tau}+\text { c.c. }
$$

where c.c. denotes the complex conjugate, $U(x, y)$ is the transverse field distribution supported by SOA cavity geometry, $\hat{e}$ is the polarization vector of the electric field and $A_{m}(z)$ are the complex amplitude (the phase matching are not included here due to very little effects).

The general case may be found for $M$ channels system by considering a WDM system with $M$ channels, where $M-1$ channels will be generated on each left and right side of the active channels. Hence, the total channels in this system will become $3 M-2$. The indices of active channels are $1,2,3, \ldots \ldots . . ., M$, while the indices of the left and right products, respectively, are $-M+2,-M+3, \ldots, 0$ and $M+1, M+2, \ldots, 2 M-1$. It is important to note that, all channels have the same pulse shape and power, such that the approximations of the pump-probe configuration cannot be done in the present problem. However, assuming the central pulsation at $w_{1}$, Eq.(1) may be rewritten as

$$
\vec{E}(r, \tau)=\hat{e} U(x, y) A(z, \tau)+c . c .
$$

where

$$
A(z, \tau)=\sum_{\ell=-M+2}^{2 M-1} A_{\ell}(z) e^{-i w_{\ell} \tau}
$$

The power of the electrical field can be determined by substituting Eq.(3) into $P(z, \tau)=|A(z, \tau)|^{2}$ to get

$$
\begin{aligned}
& P(z, \tau)=\bar{P}(z)+\Delta P(z, \tau) \\
& =\sum_{m=-M+2}^{2 M-1}\left|A_{m}(z)\right|^{2}+\sum_{\substack{m, n=-M+2 \\
m \neq n}}^{2 M-1} A_{m}(z) A_{n}^{*}(z) e^{-i(m-n) \Omega \tau}
\end{aligned}
$$

where $\Omega$ is the frequency detuning. The first term represents the total stationary optical power of all pulses. Moreover, Eq.(4) may be reformed to obtain

$$
P(z, \tau)=\sum_{m=-M+2}^{2 M-1}\left|A_{m}(z)\right|^{2}+\sum_{\ell=-3 M+3, \ell \neq 0}^{3 M-3} \Delta P_{\ell} e^{-i \ell \Omega \tau}
$$

where

$$
\Delta P_{\ell}=\sum_{m, n=-M+2, \ell=m-n}^{2 M-1} A_{m}(z) A_{n}^{*}(z)
$$

The propagation equation of an optical field in the SOA is described by the following equation [14]

$$
\frac{\partial A(z, \tau)}{\partial z}=\frac{1}{2}[(1-i \beta) g-\alpha] A(z, \tau)
$$

Eq.(7) may be modified to include the intraband and interband effects as follows

$$
\frac{\partial A(z, \tau)}{\partial z}=\frac{1}{2}\left[\sum_{\substack{m=c d p, \\ \text { shb,ch }}}\left(1-i \beta_{m}\right) g_{m}-\alpha\right] A(z, \tau)
$$

where $A$ is the pulse amplitude and $\alpha$ is the attenuation factor. The parameters $\beta_{m}$ and 
$g_{m}(m=c d p, s h b, c h) \quad$ represent the linewidth enhancement factors and the gains of the respective process. Note that, the approximation $\Delta k \approx 0$ (phase matching condition) is satisfied through the derivation of the above equations due to short device length and low dispersion of the SOA.

\subsection{Gain Dynamics}

The carrier density of electrons $N$ in the active region can be found by solving the rate equation [2]

$$
\frac{\partial N}{\partial \tau}=\frac{I}{q V}-\frac{N}{\tau_{c}}-\frac{g(N)|A|^{2}}{\tau_{c} P_{\text {sat }}}
$$

where $I$ is the current flowing through the active region of volume $V, q$ is the electron charge, $\tau_{c}$ is the carrier lifetime and $P_{\text {sat }}$ is the saturation power. The gain $g(N)$ is assumed to vary linearly with the carrier density, that is

$$
g(N)=\Gamma a\left(N-N_{o}\right)
$$

where $\Gamma$ is the confinement factor, $a$ is the gain coefficient and $N_{o}$ is the carrier density at which the active region becomes transparent. For pump-probe configuration system, Eqs.(9) and (10) will give the following solution [9]

$$
g_{c d p}=\bar{g}\left[1-\sum_{\ell=-2}^{2} \frac{\varepsilon_{c d p} \Delta P_{\ell} e^{-i \ell \Omega \tau}}{\left(1-i \ell \Omega \tau_{c d p}\right)}\right]
$$

where

$$
\begin{aligned}
& \bar{g}=\frac{g_{o}}{1+\bar{P} / P_{s a t}}, \\
& \tau_{c d p}=\frac{\tau_{c}}{1+\bar{P} / P_{s a t}}, \\
& \varepsilon_{c d p}=\frac{1}{\bar{P}+P_{s a t}}
\end{aligned}
$$

Here, $g_{o}$ represents the small signal gain. Depending on Eq.(6), the solution in Eq.(11) may be extended to any system of channels to obtain

$$
g_{c d p}=\bar{g}\left[1-\sum_{\ell=-3 M+3}^{3 M-3} \frac{\varepsilon_{c d p} \Delta P_{\ell} e^{-i \ell \Omega \tau}}{\left(1-i \ell \Omega \tau_{c d p}\right)}\right]
$$

On the other hand, the simplified differential equations for the gain coefficients of intraband effects are [14]

$$
\frac{\partial g_{m}}{\partial \tau}=-\frac{g_{m}}{\tau_{m}}-\frac{g_{s a t} \varepsilon_{m} P(z, \tau)}{\tau_{m}}
$$

where $g_{\text {sat }}$ is the saturation gain and $m=s h b, c h$. The parameters $\varepsilon_{m}$ and $\tau_{m}$ are the gain compression factors and the characteristic times of the respective process. For pump-probe configuration system, the solution of Eq.(13) will take the following form [13]

$$
g_{m}=-\bar{g}\left[\varepsilon_{m} \bar{P}(z)+\varepsilon_{m} \sum_{\ell=-2}^{2} \frac{\Delta P_{\ell} e^{-i \ell \Omega \tau}}{1-i \ell \Omega \tau_{m}}\right]
$$

Also, Eq.(14) may be extended to any number of channels depending on (6) to obtain

$$
g_{m}=-\bar{g}\left[\varepsilon_{m} \bar{P}(z)+\varepsilon_{m} \sum_{\ell=-3 M+3}^{3 M-3} \frac{\Delta P_{\ell} e^{-i \ell \Omega \tau}}{1-i \ell \Omega \tau_{m}}\right]
$$

\subsection{Generalization of Propagation Equation}

Inserting Eqs.(12) and (15) into (7), using (3) and expanding the summation of amplitudes, yields

$$
\begin{aligned}
& \frac{d A_{-(M-2)}}{d z} e^{i(M-2) \Omega \tau}+. .+\frac{d A_{-1}}{d z} e^{i \Omega \tau}+\frac{d A_{0}}{d z}+\frac{d A_{1}}{d z} e^{-i \Omega \tau}+. . \\
& +\frac{d A_{(2 M-1)}}{d z} e^{-i(2 M-1) \Omega \tau}=\frac{\bar{g}}{2}\left[\left(1-i \beta_{c d p}\right)-\sum_{m=s h b, c h} \varepsilon_{m}\left(1-i \beta_{m}\right) \bar{P}-\frac{\alpha}{2}\right] \\
& \times\left[A_{-(M-2)} e^{i(M-2) \Omega \tau}+\ldots . .+A_{-1} e^{i \Omega \tau}+\ldots .+A_{2 M-1} e^{-i(2 M-1) \Omega \tau}\right] \\
& \left.-\frac{\bar{g}}{2} \sum_{\substack{m=c d p \\
\text { shb,ch }}} \gamma_{m}\left\{\frac{\Delta P_{-(3 M-3)} e^{(3 M-3) i \Omega \tau}}{1+(3 M-3) i \Omega \tau_{m}}+\ldots .+\frac{\Delta P_{3 M-3} e^{-(3 M+3) i \Omega \tau}}{1-(3 M-3) i \Omega \tau_{m}}\right\}\right]
\end{aligned}
$$$$
\times\left[A_{-(M-2)} e^{i(M-2) \Omega \tau}+. .+A_{-1} e^{i \Omega \tau}+\ldots .+A_{2 M-1} e^{-i(2 M-1) \Omega \tau}\right]
$$

where $\gamma_{m}=\varepsilon_{m}\left(1-\beta_{m}\right)$. Eq.(16) contains a large number of terms that may be classified into $3 M-2$ differential equations depending on the exponential function at each set. However, equating the coefficients of the same exponential functions $e^{i K \Omega \tau}$, where $K$ is an integer, will generate $3 M-2$ differential equations that may be described by the generalized equation

$$
\frac{d A_{j}}{d z}=\frac{\bar{g}}{2}\left\{\Phi A_{j}-\sum_{\substack{m=c d p \\ \text { shb,ch }}} \gamma_{m}\left[\sum_{\substack{k=1 \\ k \neq 2 M-j}}^{3 M-2} \frac{\Delta P_{k-2 M+j} A_{2 M-k}}{1-i(k-2 M+j) \Omega \tau_{m}}\right]\right\}
$$

where

$$
\Phi=\left(1-i \beta_{c d p}\right)-\sum_{m=s h b, c h} \varepsilon_{m}\left(1-i \beta_{m}\right) \bar{P}-\alpha / 2
$$


and $j$ is an integer that denotes the channel index, i.e. channel location, and $M$ denotes the number of active channels in the system. The coefficient $\Delta P_{n}$ is defined as

$$
\Delta P_{n>0}=\sum_{m=-M+n+2}^{2 M-1} A_{m} A_{m-n}^{*}=\left[\Delta P_{n<0}\right]^{*}, n \neq 0
$$

Eqs.(17) and (18) are our main result in this paper. They describe how the complex amplitude of each channel evolves along amplifier by including the effects of both interband and intraband processes. The former contribution becomes negligible for $\Omega \tau_{c d p} \gg 1$ since carriers cannot respond fast enough to changes in the amplitude if $\Omega$ exceeds much more than $\tau_{c d p}^{-1}$. In general, the indices $j$ and $k$ in Eq.(17) take integer values which extends beyond the number of channels $M$ since FWM creates new frequency components on both sides of the boundary channels.

Practically, it is often sufficient to consider only $M-1$ additional channels on each side. One then solves Eq.(17) numerically with $j=-(M-1)$ to $j=2 M-1$ by setting $A_{j}(0)=0$ for values of $j$ outside the range $j=1,2,3, \ldots . M$ (active channels). Since $A_{j}$ is complex, one needs to calculate $6 M-6$ of the parameters $\Delta P_{j}$ that may be determined to form a set of $3 M-2$ nonlinear differential equations that may solve to characterize the amplifier.

As an example, the extraction of Eqs.(17) and (18) for $M=6$ and $j=4$ will give

$$
\frac{d A_{4}}{d z}=\frac{\bar{g}}{2} \Phi A_{4}-\frac{\bar{g}}{2} \sum_{m=c d p, s h b, c h} \gamma_{m} \sum_{k=1, k \neq 8}^{16} \frac{\Delta P_{k-8} A_{12-k}}{1-i(k-8) \Omega \tau_{m}}
$$

and

$$
\Delta P_{n>0}=\sum_{m=-4+n}^{11} A_{m} A_{m-n}^{*}=\left[\Delta P_{n<0}\right]^{*}, n=-7, . .,-1,1, . .8
$$

For a larger system, i.e. large $M$, the above equations will be very huge. For example if $M=20$, there is a set of $58 \times 2$ from $\Delta P_{j}$ that must be calculated to explain the propagation equations of the system. However, the analytical solution for $M>2$ is impossible, hence the numerical treatments will be adopted.

\section{Results and Discussion}

To compute the FWM performance of SOA in WDM systems, a computer program has been written in Mat Lab to analysis the system of equations in Eq.(17) using a fourth-order Rung-Kutta method with a fixed step size. The simplified computation is equivalent to dividing the SOA into $k$ sections, each of length $\Delta L=L / k$ which is equal to the step-size of the RungKutta integration. The device parameters used in the simulation are listed in table(1).

Fig.(1) represents the sidebands power as a function of channel 1 power in the case of constant power at channel 2 for 2-channel WDM system using different frequency spacing. For 2-channel system with $P_{1}>P_{2}$, any frequency spacing gives a higher conversion efficiency for the left sideband power in comparison with the right sideband. Physically, two photons at $w_{1}$ are annihilated to create two photons at $w_{1}-\Omega$ and $w_{1}+\Omega$. Similarly, two photons at the frequency $w_{2}$ are annihilated to create two photons at the frequency at $w_{2}-\Omega$ and $w_{2}+\Omega$. As a result, the left FWM product is the larger one for any frequency spacing due to $P_{1}>P_{2}$, see [9]. The situation is changed for comparable $P_{1}$ and $P_{2}$, where the saturation effects will dominate at the higher frequency. In turn, the sideband powers curves will be intersected at a certain power of the channel 1 depending on the frequency detuning. The intersection take place at higher frequency detuning for higher channel 1 power. In other words, using a same power for 2-channel system will dominate the left sideband power for lower frequency detuning and the right sideband power is dominated for higher frequency spacing. The intersection point represents the equalization of asymmetric amplification in SOA and saturation effects, where the power of channel 1 will control the shifting of intersection point.

Fig.(2) explains the behavior of powers for three WDM systems (2-channel, 4-channel and 8-channel). The figure represents the power behavior of active channels and their sum as a function of frequency detuning. The power behavior of active channels and their sum variations is well expected, where the effect of FWM is to increase the gain for the low frequency channel and to decrease it for the high frequency channel. The curve of active channels sum has approximately a constant value in higher frequency detuning and it decrease in low frequency because the FWM that causes the depletion of active channels. Generally, all active channels experience a high variation in the low frequency detuning but the variation is very small for the higher frequency detuning. Again, this situation may be attributed to the small conversion efficiency for higher frequency detuning. 


\section{Conclusions}

In conclusion, the effect of FWM on the active channels is to increase the gain for the low-frequency channel and to decrease it for the high-frequency channel. On the other hand, the effect of FWM on the sideband channels are controlled by the equivalence of the saturation effects and the asymmetry of gain, where the left and right sideband powers are varied depending on the input power and frequency detuning. For large frequency detuning, the power variations are very little, but the need for large bandwidth in optical communication will prevent this selection. As a result, we cannot avoid the FWM in WDM system.

\begin{tabular}{|l|l|l|l|}
\hline symbol & Value & symbol & Value \\
\hline$\Gamma$ & 0.4 & $\varepsilon_{\text {shb }}$ & $0.91 \mathrm{~W}^{-1}$ \\
\hline$\alpha$ & $3500 \mathrm{~m}^{-1}$ & $\varepsilon_{c h}$ & $1.62 \mathrm{~W}^{-1}$ \\
\hline$I$ & $40 \mathrm{~mA}$ & $\tau_{\text {shb }}$ & $0.036 \mathrm{ps}$ \\
\hline$V$ & $2.83 \times 10^{-16} \mathrm{~m}^{3}$ & $\tau_{c h}$ & $0.52 \mathrm{ps}$ \\
\hline$P_{\text {sat }}$ & $10 \mathrm{~mW}$ & $\beta_{c d p}$ & 10 \\
\hline$T_{o}$ & $10 \mathrm{ps}$ & $\beta_{\text {shb }}$ & 0.21 \\
\hline$P_{\text {in }}$ & $0.02 \mathrm{~mW}$ & $\beta_{c h}$ & 2.81 \\
\hline$L$ & $0.5 \mathrm{~mm}$ & $A$ & $3.9 \times 10^{9} \mathrm{~s}^{-1}$ \\
\hline$N_{o}$ & $1.6355 \times 10^{23} \mathrm{~m}^{-3}$ & $B$ & $8.5 \times 10^{-16} \mathrm{~m}^{3} / \mathrm{s}$ \\
\hline$\tau_{c}$ & $250 \mathrm{ps}$ & $C$ & $3.3 \times 10^{-40} \mathrm{~m}^{6} / \mathrm{s}$ \\
\hline
\end{tabular}

Table 1. typical parameters value of $\operatorname{InGaAsP}$ around $1550 \mathrm{~nm}[11,14]$.
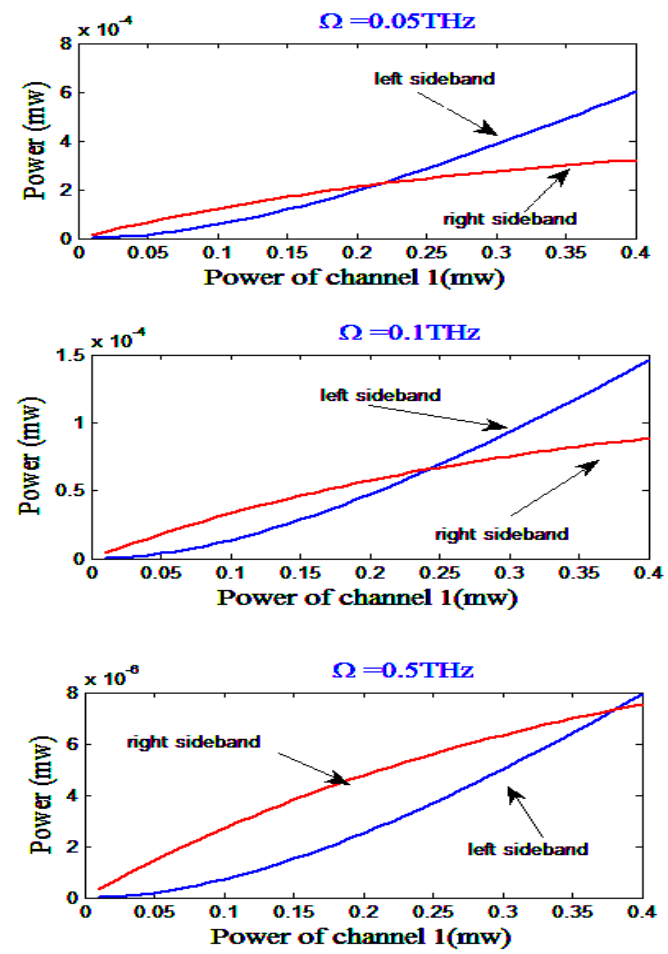

Figure 1. sidebands power (in a system of two active channels where channel 2 is constant, $P_{2}=0.2 \mathrm{~mW}$ ) as a function of power of channel 1 , for different frequency spacing.
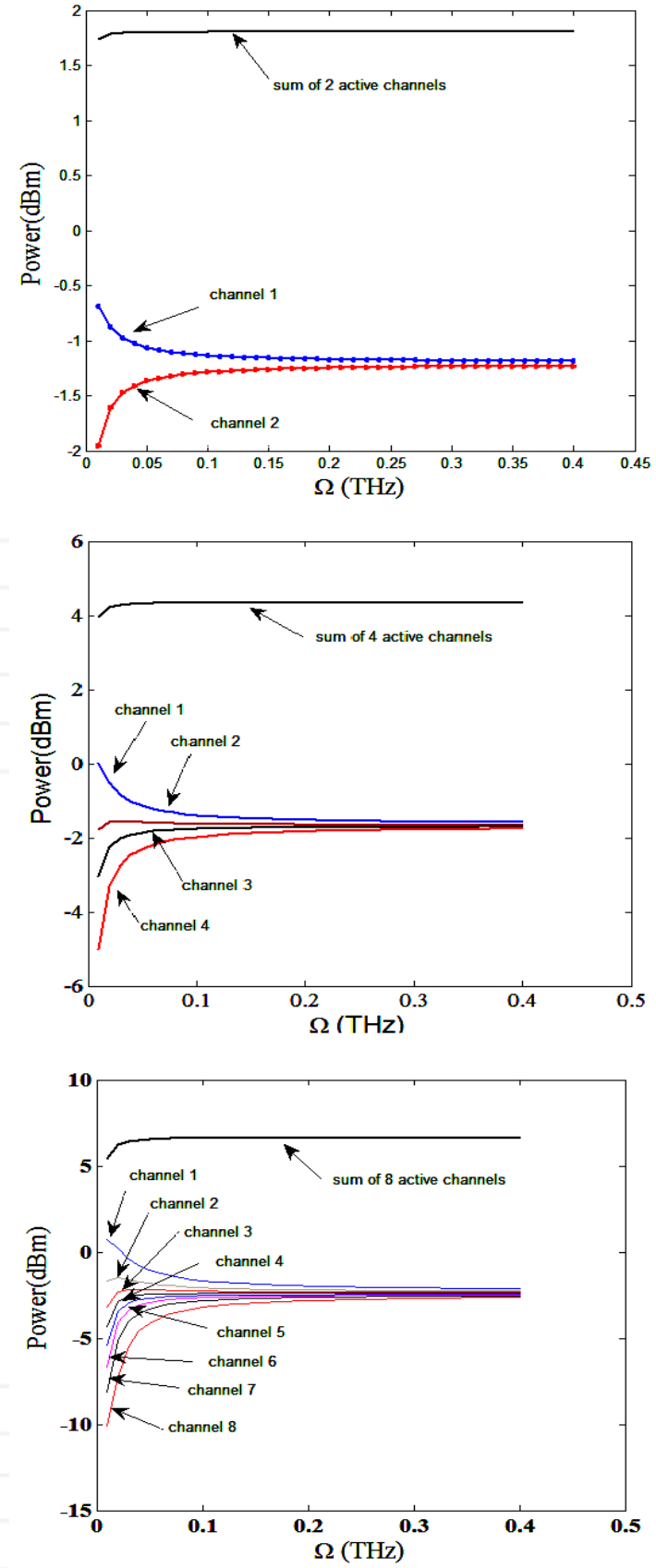

Figure 2. three WDM systems (2-channels, 4-channels, and 8channels). The power of each active channel and the sum as a function of frequency detuning.

\section{References}

[1] J. Zhou, N. Park, J. W. Dawson, K. J. Vahala, M. A. Newkirk, and I. Miller, Barry, "Efficiency of broadband four-wave mixing wavelength conversion using semiconductor traveling-wave amplifiers," IEEE Photonics Technology Letters, vol. 6, no. 1, pp. 50-52, 1994.

[2] G. P. Agrawal, "Population pulsations and nondegenerate four wave mixing in semiconductor lasers and amplifiers," J. Opt. Soc. Amer. B, vol. 5, pp. 147-159, 1988. 
[3] A. Mecozzi, S. Scotti, D. D'Ottavi, E. Iannone, and P. Spano, "Four wave mixing in traveling-wave semiconductor amplifiers," IEEE Quantum Electron., vol. 31, pp. 689-699, 1995.

[4] I. Koltchanov, S. Kindt, K. Petermann, S. Diez, R. Ludwig, R. Schnabel, and H. G. Weber, "Gain dispersion and saturation effects in four-wave mixing in semiconductor laser amplifiers," IEEE J. Quantum Electron., vol. 32, pp. 712-720, 1996.

[5] N. Schunk, "All-optical frequency conversion in a traveling-wave semiconductor laser amplifier," IEEE J. Quantum Electron., vol. 27, pp. 1271-1279, 1991.

[6] G. P. Agrawal, "Four wave mixing and phase conjugation in semiconductor laser media," Opt. Lett., vol. 12, pp. 260-262, 1987.

[7] A. Uskov, J. Mørk, and J. Mark, "Wave mixing in semiconductor laser amplifiers due to carrier heating and spectral-hole burning," IEEE J. Quantum Electron., vol. 30, pp. 1769-1781, 1994.

[8] G. Talli and M. J. Adams, "Gain dynamics of semiconductor optical amplifiers and threewavelength devices," IEEE J. Quantum Electron., vol. 39, pp. 1305-1313, 2003.

[9] G. P. Agrawal and I. M. I. Habbab, “Effect of fourwave mixing on multichannel amplification in semiconductor laser amplifiers," IEEE J. Quantum Electron., vol. 26, no. 3, pp. 501-505, 1990.
[10] C. Xie, P. Ye, and J. Lin, " Four-wave mixing between short optical pulses in semiconductor optical amplifiers with the consideration of fast gain saturation", IEEE Photon. Technol. Lett., vol. 11 No.5,1999.

[11] C. Politi, D. Klonidis, and M. Mahony, " Waveband converters based on four-wave mixing in SOAs", Lightwave Tech., Vol. 24, No. 3, 2006.

[12] A. Mecozzi and J. Mørk, "Saturation effects in nondegenerate four wave mixing between short optical pulses in semiconductor laser amplifiers," IEEE J. Sel. Topics Quantum Electron., vol. 3, no. 5, pp. 1190-1207, Oct. 1997.

[13] M. A. Summerfield and R. S. Tucker, "Frequencydomain model of multi wave mixing in bulk semiconductor optical amplifiers," IEEE J. Sel. Topics Quantum Electron, vol. 5, no. 3, pp. 839849, May/Jun. 1999.

[14] P. Runge "Nonlinear effects in ulralong semiconductor optical amplifier for optical communications: physics and applications," Ph. D. Thesis, Berlin University, 2010.
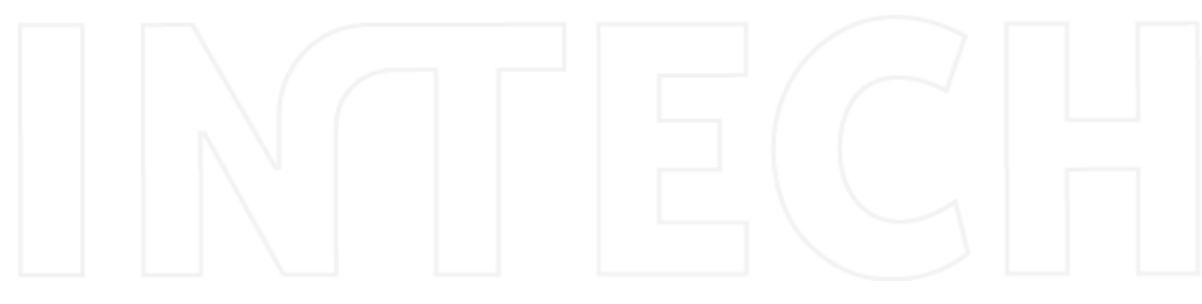